\title{
TEST OF THE INVERSE SQUARE LAW THROUGH PRECESSION OF ORBITS
}

\author{
N.I. Kolosnitsyn and V.N.Melnikov \\ Center for Gravitation and Fundamental Metrology, VNIIMS \\ and Institute of Gravitation and Cosmology, \\ Peoples' Friendship University of Russia, \\ 3-1, M.Ulyanovoi Str., 119313, Moscow, Russia \\ e-mail: melnikov@rgs.phys.msu.su
}

\begin{abstract}
Using precession of orbits due to non-Newtonian interaction between two celestial bodies and modern tracking data of satellites, planets and a pulsar we obtain new more precise limits on possible Yukawa-type deviations from the Newton law in planets (satellites) radii ranges.
\end{abstract}

\section{Non-Newtonian interactions, or range variations of G.}

Many modified theories of gravity and unified theories [1,2,3] predict some deviations from the Newton law (inverse square law, ISL) or composition-dependent violations of the Equivalence Principle (EP) due to appearance of additional fields or new possible massive particles (partners) $[5,6]$. Experimental data limit the existence of these effects at nearly all ranges at some level, but the most poor data are available at less than millimeter and also at meters and hundreds of meters ranges. The only positive result of existence of such deviation in the range of 20 to $500 \mathrm{~m}$ was obtained by Achilli et al. using an energy storage plant experiment with gravimeters. They found the deviation from the Newton law with the Yukawa potential strength $\alpha$ between 0.13 and 0.25 . This result contradicts other experimental data and needs to be verified in similar or other independent experiments, probably in space ones $[6,4,17]$.

In the Einstein theory $G$ is a true constant. But, if we think that $G$ may vary with time $[3,5]$, then, from a relativistic point of view, it may vary with distance as well. In GR massless gravitons are mediators of the gravitational interaction, they obey second-order differential equations and interact with matter with a constant strength G. If any of these requirements is violated or we consider quantum corrections to the classical theory, we come in general to deviations from the Newton law with range (or to generalization of GR).

In [6] several classes of such theories were analysed:

1. Theories with massive gravitons like bimetric ones or theories with a $\Lambda$-term.

2. Theories with an effective gravitational constant like the general scalar-tensor ones [5].

3. Theories with torsion.

4. Theories with higher derivatives (4th-order equations etc.), where massive modes in a propagator appear leading to short-range additional forces.

5. More elaborated theories with other mediators besides gravitons (partners), like 
supergravity, superstrings, M-theory etc.

6. Theories with nonlinearities induced by any known physical interactions (BornInfeld etc.), where an effective mass appears.

7. Phenomenological models, where the detailed mechanism of deviation is not known (fifth or other force).

In all these theories some effective or real masses appear leading to Yukawa-type deviation from the Newton law, characterized by strength $\alpha$ and range $\lambda$ or to a power law deviation (see [6]).

There exist some model-dependant estimations of these forces. The most well-known one belongs to Scherk (1979) from supergravity where the graviton is accompanied by a spin-1 partner (graviphoton) leading to an additional repulsion. Other models were suggested by Moody and Wilczek (1984) - introduction of a pseudo-scalar particle leading to an additional attraction between macro-bodies with the range $2 \cdot 10^{-4} \mathrm{~cm}$ $<\lambda<20 \mathrm{~cm}$ and strength $\alpha$ from 1 to $10^{-10}$ in this range. Another supersymmetric model was elaborated by Fayet $(1986,1990)$, where a spin-1 partner of a massive graviton gives an additional repulsion in the range of the order $10^{3} \mathrm{~km}$ and $\alpha$ of the order $10^{-13}$.

A scalar field to adjust $\Lambda$ was introduced also by $\mathrm{S}$. Weinberg in 1989, with a mass smaller than $10^{-3} \mathrm{eV} / \mathrm{c}^{2}$, or a range greater than $0.1 \mathrm{~mm}$. One more variant was suggested by Peccei, Sola and Wetterich (1987) leading to additional attraction with a range smaller than $10 \mathrm{~km}$. Some p-brane models also predict non-Newtonian additional interactions, in particular in the mm range, what is intensively discussed nowadays in the hierarchy and braneworld models. About PPN parameters for multidimensional models with p-branes see $[8]$.

In this paper we consider limits on a possible Yukawa type additional interaction in the ranges of the order of planets (satellites) distances from the Sun (the Earth) using the precession method and satellites plus planets tracking data.

\section{Basic formulas.}

As it is known, in two bodies problem an orbit of a celestial body is closed for only two interaction potentials [9]. They are:

(1) the Newtonian potential: $U \sim 1 / r$ and

(2) $U \sim r^{2}$.

In other cases the orbit is not closed and a pericenter precession is observed. In particular, the deviation from the Newton law due to the Yukawa additional interaction

$$
U^{\prime}=\frac{G m_{1} m_{2}}{r} \alpha \exp (-r / \lambda)
$$

entails a precession of an orbit.

In a general case the precession magnitude due to a small perturbation, described by a potential $\delta U$, is equal to (see [9]) 


$$
\delta \varphi=\frac{\partial}{\partial M}\left(\frac{2 m}{M} \int_{0}^{\pi} r^{2} \delta U d \varphi\right)
$$

Integration is done over a "non-perturbed" trajectory. Here $m_{1}$ is a mass of one celestial body, $m_{2}$ is the mass of another celestial body, $M=m r^{2} \dot{\varphi}$ is the integral of motion (the angular momentum), $m=m_{1} m_{2} /\left(m_{1}+m_{2}\right)$ is the reduced mass,

$\delta U=\alpha\left(G m_{1} m_{2} / r\right) \exp (-r / \lambda)$.

The "non-perturbed" trajectory is described by the expressions:

$$
r=\frac{p}{1+e \cos \varphi}, \quad e^{2}=1+\frac{2 E M^{2}}{m\left(G m_{1} m_{2}\right)^{2}}, \quad p=\frac{M^{2}}{m G m_{1} m_{2}}=a\left(1-e^{2}\right) .
$$

where $e$ is an eccentricity, $a$ is a semi-major axis.

After differentiating the right-hand side of Eq.(2) with respect to $\mathrm{M}$ we obtain

$$
\delta \varphi=2 m G m_{1} m_{2} \alpha\left\{-\frac{1}{M^{2}} \int_{0}^{\pi} r e^{-r / \lambda} d \varphi+\frac{1}{M} \int_{0}^{\pi}\left(1-\frac{r}{\lambda}\right) e^{-r / \lambda} \frac{\partial r}{\partial M} d \varphi\right\}
$$

Taking into account Eq. (3) we get

$$
\frac{\partial r}{\partial M}=\frac{r}{M}\left[1+\frac{e+\cos \varphi}{e(1+e \cos \varphi)}\right]
$$

After substitution of (5) in (4) we obtain

$$
\delta \varphi=2 \alpha \frac{m G m_{1} m_{2}}{M^{2}} \int_{0}^{\pi} r e^{-r / \lambda}\left[-\frac{e+\cos \varphi}{e(1+e \cos \varphi)}+\frac{r}{\lambda}\left(1+\frac{e+\cos \varphi}{e(1+e \cos \varphi)}\right)\right] d \varphi
$$

As a result, we have

$$
\delta \varphi=\alpha \frac{2}{e} \int_{0}^{\pi} \frac{\exp (-r / \lambda)}{(1+e \cos \varphi)^{2}}\left\{\frac{r}{\lambda}\left[2 e+\left(1+e^{2}\right) \cos \varphi\right]-(e+\cos \varphi)\right\} d \varphi
$$

where

$$
\frac{r}{\lambda}=\frac{a}{\lambda} \frac{1-e^{2}}{(1+e \cos \varphi)}
$$

\section{Estimation of effects.}


Using Eq. (7) and proposed data on $\delta \varphi$ error for the LAGEOS Satellites, the inner planets, Moon and the binary pulser B1913+16, we calculated curves $\alpha(\lambda)$, which determine a bound in the plane $(\alpha, \lambda)$ between two domains, where the Yukawa interaction (a new nonnewtonian force) is forbidden by experiment and where it is not. The sensitivities to Yukawa interactions - the united curve $\alpha(\lambda)$ described by Eq.(7) - are shown in Fig.1 as the domain 1998 for the parameter $\lambda$ in the range from $1 \cdot 10^{6} \mathrm{~m}$ to $1 \cdot 10^{13} \mathrm{~m}$.

We took data for LAGEOS and LAGEOS II from [11] related to the relativistic LenseThirring precession. The data contain information on classical (Newtonian) and relativistic precession. The last one equals to $\sim 57$ milliarcseconds per year for LAGEOS II. The error for test of the Lense-Thirring effect is equal to $20 \%$. Therefore, we took the error $\delta \varphi=11.4$ milliarcseconds per year. The LAGEOS II eccentricity $e=0.014$. Using the data we obtained $\alpha_{\min }=1.38 \cdot 10^{-11}$ for $\lambda=6.081 \cdot 10^{6} \mathrm{~m}$. Our result is in a very good agreement with the estimation for $\alpha \sim 1 \cdot 10^{-11}$ obtained by Iorio (see [20].

For the Moon there are high precision data of Laser Ranging (see, for example [12]). In Ref. [12] geodetic precession (first calculated by de Sitter), equaled to $19 \mathrm{~ms}$ per year, was determined with the error $0.9 \%$. The absolute error was equal to $1,71 \cdot 10^{-4} " / y$. We used this estimation and obtained $\alpha_{\min }=3.64 \cdot 10^{-11}$ for $\lambda=1,92 \cdot 10^{8} \mathrm{~m}$.

Very precise information on the Mercury precession is contained in the article of Pitjeva [13]. Using radar observations of Mercury for the time span 1964-1989 and taking its topography Pitjeva has estimated systematic errors for the perihelion motion and a new random error for this motion as $0.052 " / c y$. From this it follows that $\alpha_{\min }=3.57 \cdot 10^{-10}$ for $\left(\lambda=2.89 \cdot 10^{10} \mathrm{~m}\right)$.

The most precise data on the Mars precession are obtained from observations of the landing spacecraft Pathfinder (see [14] ). Pitjeva has analysed joint observation data of Viking and Pathfinder missions and has obtained more precise estimation for precession of the Mars [19]. However a sensitivity to the Yukawa force in this case is very small and the sensitivity curve for Mercury dominates.

We also used data for the binary pulsar PSR $1913+16$. The pulsar has an orbit with the high eccentricity $e=0.617$ and a big precession $4,23^{\circ} / y \pm 0,000007^{\circ} / y$. Using parameters of the pulsar in [15] we calculated $\alpha_{\min }=6.409 \cdot 10^{-11}$ for $\lambda=7.515 \cdot 10^{8} \mathrm{~m}$.

Fig. 1 shows our data (LAGEOS, MOON, PSR1913, MERCURY) as the new experimental constraint on the coupling parameter $\alpha$ as a function of the parameter $\lambda$ taken from the reference [16].

\section{Conclusions}

Here, we presented some new limits on possible deviations from the inverse square law using planets and satellite tracking data. They improve the existing ones by several orders at satellite $\left(\sim 6 \cdot 10^{6} \mathrm{~m}\right)$ and planets distance from the Sun $\left(\sim 1 \cdot 10^{13} \mathrm{~m}\right)$ ranges. Some proposed space projects (such as SEE) make possible to improve these estimations to five orders at a meter range (see [17] and [10] ). Limits at less than mm ranges see in [18]. 


\section{References}

[1] V.N. Melnikov, "Multidimensional Classical and Quantum Cosmology and Gravitation. Exact Solutions and Variations of Constants." CBPF-NF-051/93, Rio de Janeiro, 1993, 93pp.;

V.N. Melnikov, in: "Cosmology and Gravitation", ed. M. Novello, Editions Frontieres, Singapore, 1994, p. 147.

[2] V.N. Melnikov, "Multidimensional Cosmology and Gravitation", CBPF-MO-002/95, Rio de Janeiro, 1995, 210 pp.;

V.N. Melnikov. In: Cosmology and Gravitation. II, ed. M. Novello, Editions Frontieres, Singapore, 1996, p. 465.

[3] K.P. Staniukovich and V.N. Melnikov, "Hydrodynamics, Fields and Constants in the Theory of Gravitation", Energoatomizdat, Moscow, 1983, 256 pp. (in Russian).

V.N.Melnikov, "Fields and Constants in the Theory of Gravitation", CBPF-MO02/02, Rio de Janeiro, 2002, 145 pp.

[4] V.N.Melnikov, "Gravity as a Key Problem of the Millennium", Proc.2000 NASA/JPL Conf. Fund. Phys. in Microgravity, Solvang, 2000, NASA Doc. D-21522, 2001, pp. 4.1-4.17, gr-qc/0007067.

[5] V.N. Melnikov, Int. J. Theor. Phys. 33, 1569 (1994).

[6] V. de Sabbata, V.N. Melnikov and P.I. Pronin, Prog. Theor. Phys. 88, 623 (1992).

[7] V.N. Melnikov. In: "Gravitational Measurements, Fundamental Metrology and Constants", eds. V. de Sabbata and V.N. Melnikov, Kluwer Academic Publ., Dordtrecht, 1988, p. 283.

[8] V.D.Ivashchuk and V.N.Melnikov. Exact Solutions in Multidimensional Gravity with Antisymmetric Forms. Topical Review. Class. Quant.Grav., 2001, v.18, pp.R1-R66

[9] L.D. Landau and E.M.Lifshitz, Mechanics, M., Nauka, 1972.

[10] A.D.Alexeev, K.A.Bronnikov, N.I.Kolosnitsyn, M.Yu.Konstantinov, V.N.Melnikov, A.J.Sanders. Metrologia, v. 38, No 5, pp. 397-408 (2001).

[11] Ciufolini, E. Pavlis, F. Chieppa, E. Fernandes-Vieira, J. Perez-Mercarder. Science, v. 279, 2100-2103 (1998)

[12] J.O. Dickey, P.L. Bender et al. Science, v.265, 482-490 (1994).

[13] E.V.Pitjeva. Celestial Mechanics and Dynamical Astronomy, v. 55, 313-321 (1993).

[14] W.M. Folkner, C.F. Yoder, B.N. Yuan, E.M. Standish, R.A. Preston. Science, v. 278, 1749-1752 (1997).

[15] J.M. Weisberg and J.H. Taylor. astro-ph/0211217. 
[16] E. Fishbach, C. Talmage. Twelve years of the fifth force. Preprint IN7907-1396, Physics Department, Purdue University, West Lafayette, USA, 1998.

[17] Sanders and W. Deeds. Phys. Rev. D 46, 480 (1992).

[18] C.D.Hoyle et al., Phys.Rev.Lett. 86 (2001) 1418-1421.

[19] E.V. Pitjeva, Proc. IAA RAS, N4, 22-33, (1999).

[20] L. Iorio. Phys.Lett.A, 2002, 298, 315-318. 
This figure "Grafic1.jpg" is available in "jpg" format from: http://arxiv.org/ps/gr-qc/0302048v1 